Article

\title{
Burned Area Mapping in the Brazilian Savanna Using a One-Class Support Vector Machine Trained by Active Fires
}

\author{
Allan A. Pereira ${ }^{1, *}$, José M. C. Pereira ${ }^{2,+}$ (D), Renata Libonati ${ }^{3,+}$ (D), Duarte Oom ${ }^{2,+}$ (D), \\ Alberto W. Setzer ${ }^{4,+}$, Fabiano Morelli ${ }^{4,+}$, Fausto Machado-Silva ${ }^{3,+}$ (iD and \\ Luis Marcelo Tavares de Carvalho ${ }^{5,+}$ \\ 1 Instituto Federal de Ciência e Tecnologia do Sul de Minas Gerais, 37713-100 Poços de Caldas, Brazil \\ 2 Centro de Estudos Florestais, Instituto Superior de Agronomia, Universidade de Lisboa, \\ 1349-017 Lisboa, Portugal; jmocpereira@gmail.com (J.M.C.P.); duarte.oom@gmail.com (D.O.) \\ 3 Departamento de Meteorologia, Universidade Federal do Rio de Janeiro, 21941-916 Rio de Janeiro, Brazil; \\ renata.libonati@igeo.ufrj.br (R.L.); fausto.eco@gmail.com (F.M.-S.) \\ 4 Centro de Previsão do Tempo e Estudos Climáticos, Instituto Nacional de Pesquisas Espaciais, \\ 12227-010 São José dos Campos, Brazil; alberto.setzer@cptec.inpe.br (A.W.S.); \\ fabiano.morelli@cptec.inpe.br (F.M.) \\ 5 Departamento de Engenharia Florestal, Universidade Federal de Lavras, 37200-000 Lavras, Brazil; \\ passarinho@dfc.ufla.br \\ * Correspondence: allan.pereira@ifsuldeminas.edu.br; Tel.: +55-35-99188-5985 \\ $\dagger$ These authors equally contributed to this work.
}

Received: 7 September 2017; Accepted: 7 November 2017; Published: 14 November 2017

\begin{abstract}
We used the Visible Infrared Imaging Radiometer Suite (VIIRS) active fire data (375 m spatial resolution) to automatically extract multispectral samples and train a One-Class Support Vector Machine for burned area mapping, and applied the resulting classification algorithm to 300-m spatial resolution imagery from the Project for On-Board Autonomy-Vegetation (PROBA-V). The active fire data were screened to prevent extraction of unrepresentative burned area samples and combined with surface reflectance bi-weekly composites to produce burned area maps. The procedure was applied over the Brazilian Cerrado savanna, validated with reference maps obtained from Landsat images and compared with the Collection 6 Moderate Resolution Imaging Spectrometer (MODIS) Burned Area product (MCD64A1) Results show that the algorithm developed improved the detection of small-sized scars and displayed results more similar to the reference data than MCD64A1. Unlike active fire-based region growing algorithms, the proposed approach allows for the detection and mapping of burn scars without active fires, thus eliminating a potential source of omission error. The burned area mapping approach presented here should facilitate the development of operational-automated burned area algorithms, and is very straightforward for implementation with other sensors.
\end{abstract}

Keywords: support vector machine one class; burned area; active fire; Cerrado; PROBA-V; VIIRS

\section{Introduction}

Vegetation burning is a global-scale process that affects the global distribution and structure of vegetation, major biogeochemical cycles, and the climate system [1]. The relation of fire with vegetation is contradictory: as an ecological factor, it contributes to maintaining ecosystem dynamics, productivity and biodiversity and, as a land management tool, it is extensively employed in croplands, rangelands, and forests throughout the world. However, wildfires are a socio-natural hazard that annually affect millions of hectares of forests, woodlands, and other vegetation, endangering human populations, and causing substantial economic losses, both in terms of assets destroyed and in the 
form of prevention and suppression costs [2]. During the last decades, the Brazilian savanna has been increasingly affected by deforestation due to cropland and pasture expansion, consequently increasing and altering the natural fire regime in the region [3,4]. Attempts to characterize these anthropogenic impacts presuppose understanding of spatial and temporal fire patterns [1]. Despite the high frequency of human induced-fire and significant disturbance caused to the Cerrado biome, fire dynamics are not yet well characterized.

Over the last few decades, the use of remote sensing has allowed unprecedented advances in mapping fire dynamics, especially for locating fire occurrence in time and space and quantifying the total extent of area burned. Several studies relied on the use of remote sensing to map burned areas at a global/regional scale [5-11]. However, the variable persistence of burn scars within different vegetation types, and the spectral confusion with other phenomena (e.g., cloud shadowing) are some of the problems that still hamper accurate burned area mapping [12]. Accordingly, users of burned area maps have stressed the need to improve product accuracy, namely in order to refine current estimates of burned areas, thus providing input to global analysis of ecological impacts of fires to better understand the relations between fire occurrence and biodiversity, and to improve the assessment of atmospheric emissions derived from vegetation fires [13,14].

The detection of small burned areas is one of the main limitations in burned area mapping that uses low-resolution sensors, as previously reported [7,11,15-17]. It is possible to improve the detection of small burned areas using existing fire products. Alonso-Canas [5], using 300-m MEdium Resolution Imaging Spectrometer (MERIS) global satellite data, developed an algorithm based on the time series of surface reflectance to identify abrupt changes in near-infrared reflectance (NIR), and regional growing techniques using MODIS active fire as spatial seeds, in a two-phase algorithm. The use of this sensor has improved small fire detection due to better spatial resolution, however, the results were not considered competitive in comparison with MODIS burned area products [17]. Hybrid approaches that combine active fire information with reflectance data have been widely used in burned area mapping to add new evidence for burned area classification [5,7,11]. In such approaches, active fires are used to derive statistics for burn classes $[18,19]$, or used as seed points in regional growing techniques. However, active fire detection products often omit burned area patches [20], leading to underestimation of the area burned. Omission errors from active fire detection products may be due to the spatial and the temporal coverage of satellite overpasses, sensor saturation, or obscuration by clouds and smoke $[7,11,21]$. The presence of thick clouds and heavy smoke layers is a major drawback in operational applications, preventing hot spot detection due to the spectral signal attenuation in the atmosphere [21,22]. This is especially true in the tropics during the dry season [23,24], where the probability of cloud-free observations is, on average, less than $30 \%$ [25]. For instance, Schroeder [21] assessed active fire temporal continuity over Brazil using the Geostationary Operational Environmental Satellite (GOES) hotspot product and indicated that a reduction of 15\% in fire counts occurs due to cloud obscuration.

The approach described in this paper only uses active fire data to select burned pixels (positive training samples), avoiding the need to collect unburned (negative) training pixels. Collection of positive training samples can be used in a positive — only classification approach [26], and is particularly interesting for mapping burned areas since training sample collection is normally a difficult task when considering the unburned area class. However, few studies have applied this technique for burned area mapping. In a comparative study among Maximum Likelihood (ML), Binary Support Vector Machine (SVM) and One-Class Support Vector Machine Description (OC-SVDD) aimed at burned area identification, the authors of [27] reported that SVM and OC-SVDD produced good results for a broader range of sample sizes than ML. Although the SVM yields better accuracy, the authors mention as an advantage of OC-SVDD that it does not require training samples from unburned areas. However, burned area training data are manually collected, impairing its use in an automated burned area algorithm. Song et al. [28] proposed mapping burned areas applying a one-class classifier and obtained reasonable results using a kernel sparse representation model to 
represent burned samples. However, in both studies, training data for the single class of interest was collected manually, which increases the classification cost, making it dependent on human intervention for sample acquisition. Besides eliminating the subjectivity of the human intervention, the fully automatic approach is advantageous for maximizing image data processing efficiency for large area coverage and data volume in a timely and cost-effective way, enabling operational delivery of burned area products.

Here, we explore the suitability of the One-Class Support Vector Machine classifier for burned area classification and mapping in the Cerrado region and propose using active fire data to automatically collect burned area training samples, circumventing the need for human intervention and increasing the degree of automation of the entire classification procedure. The procedure was applied to $300-\mathrm{m}$ spatial resolution imagery from the Project for On-Board Autonomy-Vegetation (PROBA-V) and Visible Infrared Imaging Radiometer Suite (VIIRS) active fire data (375 m spatial resolution). This algorithm, hereafter designated AQM-PROBA (from "Área Queimada", meaning Burned Area, in Portuguese), is based on VIIRS active fire data which are used to automatically extract burned area training samples from PROBA-V reflectance imagery. Then, those samples are used as input to a one-class support vector machine classifier, which only requires positive (i.e., burned area) training data [29]. Our goal is to generate a completely automated methodology, capable of producing results with accuracy levels in the range required by users of global fire data products, such as climate and vegetation researchers, as well as land managers and policy-makers [13]. The accuracy of our results was assessed using reference data derived from Landsat-8 OLI data over the Cerrado region and finally compared with MODIS standard burned area product.

\section{Study Area and Data}

The Cerrado is the largest continuous savanna area in the world and covers about 2 million $\mathrm{km}^{2}$, ranging from $2.3^{\circ} \mathrm{S}$ to $24.7^{\circ} \mathrm{S}$ and $41.7^{\circ} \mathrm{W}$ to $60.1^{\circ} \mathrm{W}$ (Figure 1). It is one of the most important biodiversity hotspots in the world due to species richness and a high percentage of endemism, containing more than 10,000 cataloged plant species $[30,31]$, in which species display morphological and functional dependence from fire [32-34]. Several authors have shown that although fire-dependent ecosystems, such as the Cerrado, have evolved in the presence of recurrent natural fires and are dependent on them to maintain their biodiversity, high human pressure through frequent burning may have negative impacts on species diversity $[35,36]$. Fire is commonly used in agricultural land management, affecting water flow and facilitating erosive processes [37], and promoting deterioration of physical and chemical soil characteristics, reducing its productive potential [38]. Recently, the human footprint was reported as being high to very high across the Cerrado, where only a few areas remain undisturbed [39]. Moreover, current climate change scenarios point towards an overall increase in fire frequency and intensity over a large area of the Cerrado [40].

According to Koppen's classification [41], the Cerrado climate is Equatorial savanna with dry winter (Aw), characterized by dry winters and monthly mean temperatures above $18^{\circ} \mathrm{C}$. The biome has a marked dry season from May to September when the region becomes susceptible to fire events with an annual fire occurrence peak in September [7,42,43]. The region is considered a pyrobiome [33] because it is a fire-dependent ecosystem, where the majority of the species evolved in the presence of fire. According to the authors of $[7,40]$, the intra and inter-annual variabilities of fire in the Cerrado are closely related to precipitation variability, but it is worth emphasizing that human activity also plays a prominent role in fire dynamics in this region and cannot be disregarded $[33,34]$.

Two datasets were used to develop the algorithm: (1) PROBA-V near-infrared Top of Canopy data (NIR-TOC); and (2) VIIRS active fire data. The datasets span the period 1 August 2015 to 31 October 2015, corresponding to the driest months over the region $[7,40]$.

The PROBA-V satellite was launched on 6 May 2013 as a continuity mission to the Vegetation instruments aboard of Satellite Pour l'Observation de la Terre (SPOT) (1998-2014) [44]. Its orbit is sun-synchronous with an initial overpass time at about 10:45 a.m., with a $2295 \mathrm{~km}$ swath and daily 
near-global (90\%) coverage [45]. PROBA-V cameras have a spatial resolution of 100 to $180 \mathrm{~m}$ in the central $500 \mathrm{~km}$ of the swath, and 350-660 $\mathrm{m}$ along the outer sections of the swath. Final products are available at resolutions of $100 \mathrm{~m}, 300 \mathrm{~m}$, and $1 \mathrm{~km}$, with level 3 geometric and radiometric corrections. Data consist of reflectance values at the Top of the Atmosphere (product S1-TOA) and Top of Canopy (S1TOC product) in the Blue $(0.464 \mu \mathrm{m})$, Red $(0.665 \mu \mathrm{m})$, NIR $(0.837 \mu \mathrm{m})$, and shortwave infrared (SWIR; $1.603 \mu \mathrm{m}$ ) channels. PROBA-V data are disseminated by European independent research and technology organisation called VITO [46], in partnership with the European Space Agency (ESA). Solar zenith angles and viewing zenith angles data are also available, as well as information about radiometric quality and cloud/cloud shadow cover. The present study area is coincident with two PROBA-V tiles: X13Y08 and X13Y09 (Figure 1), covering an area of about 1.22 million $\mathrm{km}^{2}$ of Cerrado, which represents $60 \%$ of the entire biome area.

VIIRS active fire data, at $375 \mathrm{~m}$ spatial resolution, are used for collecting burned area spectral data training samples [47]. Active fires from VIIRS have the highest spatial resolution of currently global products, which makes them particularly suitable to detect small fires [48,49]. VIIRS was launched in October 2011 aboard the Suomi National Polar-orbiting Partnership (S-NPP) satellite, a United States mission jointly managed by the National Aeronautics and Space Administration (NASA) and by the National Oceanic and Atmospheric Administration (NOAA) and the data are provided at 12-h intervals.

Accuracy assessment is needed to quantify the degree of agreement between mapped products and ground observations. It allows for rigorous evaluation of the quality of thematic maps derived from remotely sensed data. Ideally, accuracy assessment of maps based on remotely sensed data is performed against ground measurements, taken to represent the true status or quantity of the target under analysis. There are limitations to using this ideal approach in the proposed study, due to the very broad extent of the study area (the whole of Cerrado), the limited accessibility of many regions, and the ephemeral nature of the signal, which starts to fade out a few days after the fire occurrence. Currently, this problem is circumvented by using higher spatial resolution satellite imagery as reference data for evaluating the lower resolution derived maps, being a well-established procedure $[7,15,16,18,48,50-52]$. Accordingly, the higher spatial resolution satellite imagery used in this study as reference data for accuracy assessment came from the Landsat-8 (L8) Operational Land Imager (OLI), which has a spatial resolution $(30 \mathrm{~m})$, more than 2-3 orders of magnitude higher than that of the evaluated PROBA-V and MODIS instruments. Thirteen paths/rows from OLI sensor, covering an area corresponding to $17 \%$ of the Cerrado (Figure 1), were used to elaborate independent fire reference perimeters. The procedure is based on the International Global Burned Area Satellite Product Validation Protocol [53], which uses a semi-automatic classification approach. The algorithm uses fixed and multitemporal thresholds applied to the Normalized Burn Ratio Long SWIR (NBRL) spectral index, based on OLI bands 6 and 7 (shortwave infrared around 1.6 and $2.1 \mu \mathrm{m})$. Visual photointerpretation is the ultimate benchmark for any classification and segmentation procedure [54]. Accordingly, the derived scars were then subject to a meticulously, lengthy, and expensive manual and visual quality control, in order to produce the highest quality reference map.

The choice of L8 scenes was guided by the spatial and temporal distribution of active fires derived from the VIIRS in 2015 (Figure 2). September was the month with the highest frequency of active fires, followed by October, and they are concentrated in the northern part of the Cerrado, corroborating previous studies $[7,42,43]$. L8 images were then selected according to the period of highest fire activity and lowest cloud cover. We have stratified L8 reference scenes by region according to high, medium, and low fire incidence, to properly assess commission and omission errors. Table 1 shows the path/row and dates (initial and final date) of the L8 images used in this study.

Finally, we describe our results through a comprehensive assessment of burned area classification using Landsat fire reference perimeters and comparison with the MCD64A1 collection 5 burned area product [11]. MCD64A1 uses daily reflectance and active fire data from the MODIS sensor aboard 
the AQUA and TERRA. It presents a nominal resolution of $500 \mathrm{~m}$ and is globally available on a monthly basis since August 2000. MODIS tiles h13v09 and h13v10 were used for comparison exercise; the dataset was downloaded from the University of Maryland site.

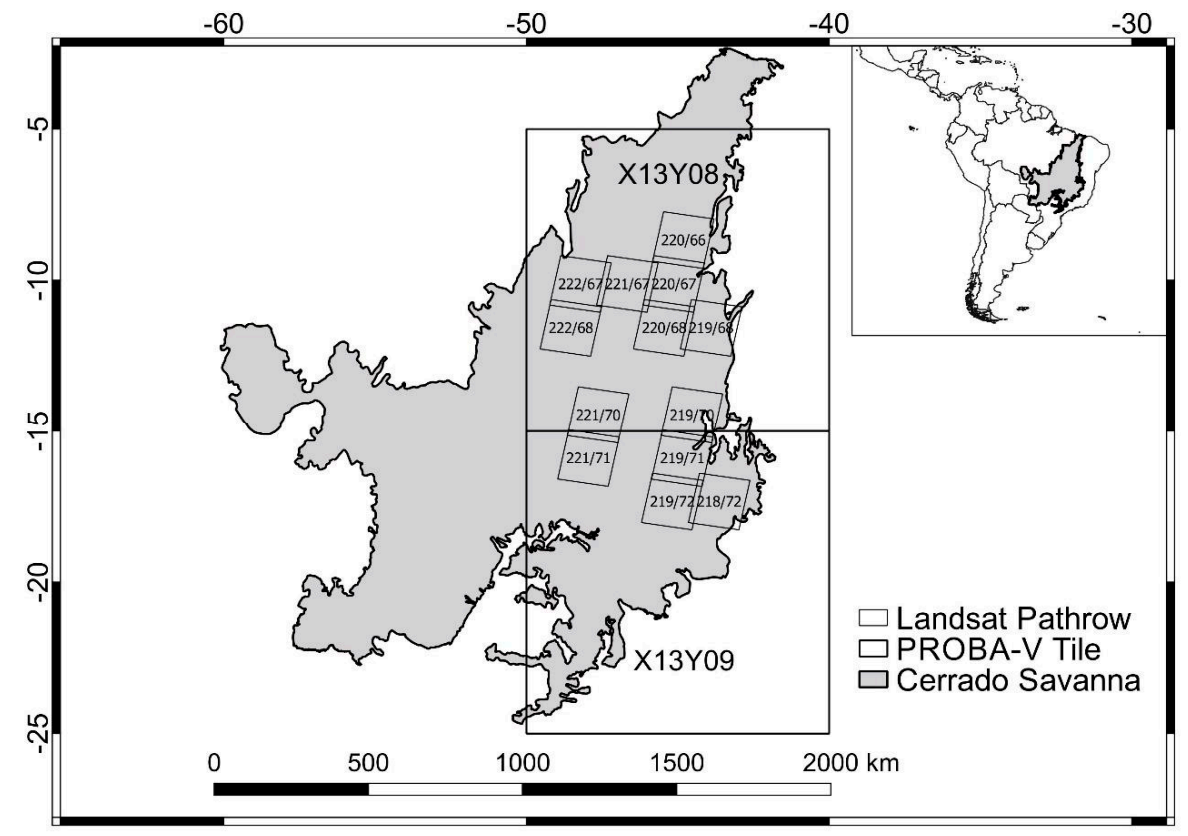

Figure 1. Location of the Cerrado savanna biome in South America (upper right), the study area (center) outlined by the two Project for On-Board Autonomy-Vegetation (PROBA-V) tiles (X13Y08/09) and the 13 Landsat 8 reference scenes (with path/rows) also shown.
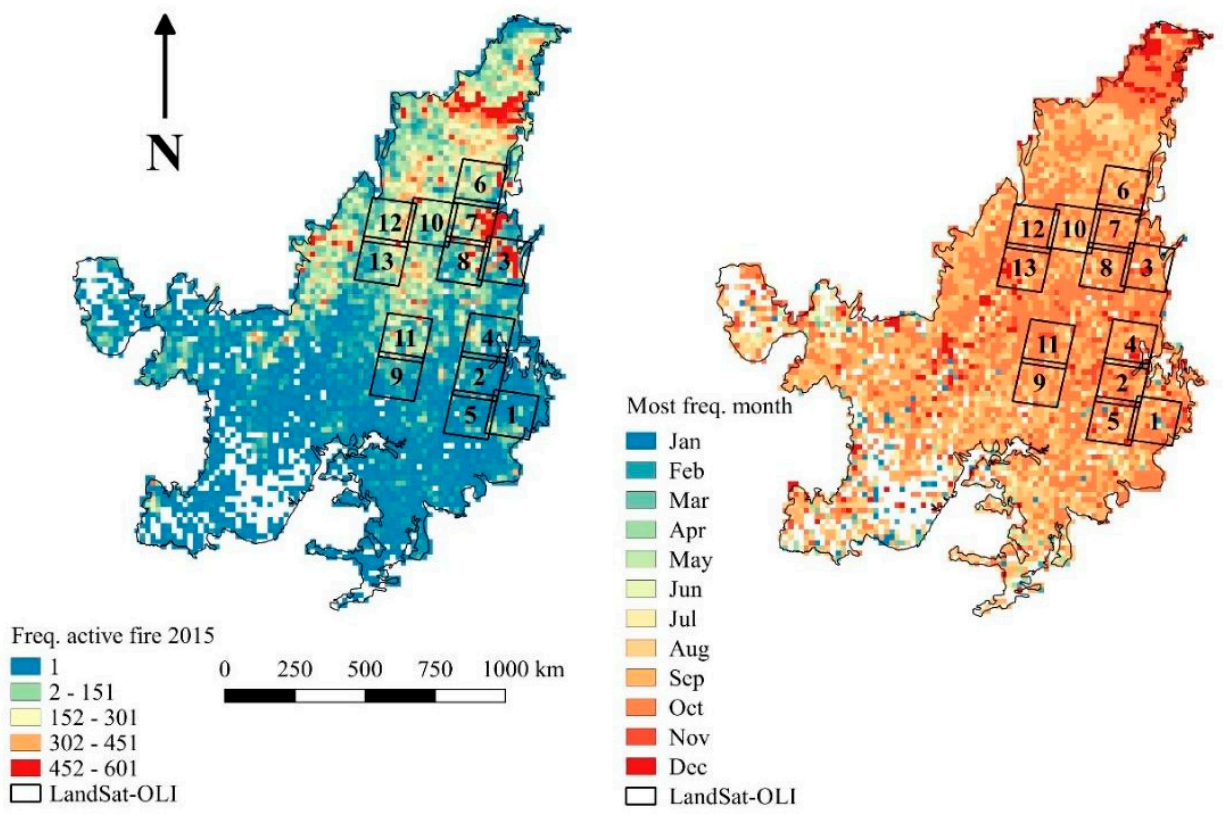

Figure 2. The frequency of Visible Infrared Imaging Radiometer Suite (VIIRS) active fires (left panel) and monthly active fires frequency (right panel) during 2015 Landsat 8 Operational Land Imager (LandSat-OLI) scene frames are also shown (black squares); numbers inside each square are depicted in Table 1. 
Table 1. Landsat 8 path/rows and dates used to elaborate reference fire perimeters.

\begin{tabular}{cccc}
\hline Number & Path/Row & Initial Date & Final Date \\
\hline 1 & $218 / 072$ & 16 September 2015 & 2 October 2015 \\
2 & $219 / 068$ & 23 September 2015 & 9 October 2015 \\
3 & $219 / 070$ & 7 September 2015 & 9 October 2015 \\
4 & $219 / 071$ & 23 September 2015 & 9 October 2015 \\
5 & $219 / 072$ & 23 September 2015 & 9 October 2015 \\
6 & $220 / 066$ & 30 September 2015 & 19 October 2015 \\
7 & $220 / 067$ & 30 September 2015 & 19 October 2015 \\
8 & $220 / 068$ & 29 August 2015 & 14 September 2015 \\
9 & $221 / 067$ & 20 August 2015 & 5 September 2015 \\
10 & $221 / 070$ & 5 September 2015 & 21 September 2015 \\
11 & $221 / 071$ & 5 September 2015 & 21 September 2015 \\
12 & $222 / 067$ & 27 August 2015 & 12 September 2015 \\
13 & $222 / 068$ & 27 August 2015 & 12 September 2015 \\
\hline
\end{tabular}

\section{Methods}

\subsection{The AQM-PROBA Algorithm}

The AQM-PROBA algorithm structure is described following main phases: Pre-processing, Multitemporal compositing, Training sample selection and Burned area classification.

\subsubsection{Pre-Processing}

PROBA-V NIR daily reflectance values, with spatial resolution of $300 \mathrm{~m}$, are georeferenced based on coordinate information contained in the metadata, rejecting pixels:

(1) containing solar zenith angles greater than $60^{\circ}$ and/or viewing zenith angles of NIR channel greater than $40^{\circ}$;

(2) classified as cloudy in the PROBA-V Quality assurance layers;

(3) containing low radiometric quality;

(4) containing reflectance values higher than 0.5 .

\subsubsection{Multitemporal Compositing}

Pixel quality assessment is provided for the product 1-day Synthesis Products Top-Of-Canopy reflectance (S1TOC). Bi-weekly composites of the second lowest NIR value of the time series were computed, with the chosen span of two weeks. Choice of this compositing approach is based on the results obtained by [55]. Multi-temporal image compositing is the creation of a synthesis image with inputs from different dates selected according to spectral criteria that minimize cloud contamination and evidence target land cover types. This approach is frequently used in burned area mapping $[7,11,56]$.

\subsubsection{Training Sample Selection}

Burned area classification mainly relies on two variables: (1) Post-fire NIR reflectance; and (2) Difference between pre-fire (T1) and postfire (T2) NIR reflectance. VIIRS active fires were used to extract NIR reflectance from PROBA-V pixels to act as training samples to the One-class Support Vector Machine (OC-SVM) classifier. A total of 323,259 VIIRS active fires (for both PROBA-V tiles) were used to extract NIR reflectance information from pixels (one active fire per pixel). As active fires may be detected by the sensor even when only a very small fraction of the pixel area has burned, its reflective spectral signature may not display clear evidence of the burning event. Hence, those pixels are inadequate training samples and have to be discarded. To exclude these samples, the set of pixels containing an active fire detected during the respective compositing period were segmented into three classes, using the Jenks natural breaks method [57]. Natural break segmentation of potential 
training data was performed on post-fire NIR reflectance values (T2) and on the pre-fire minus post-fire NIR difference values (T1-T2). To be included in the training sample, candidate pixels must belong simultaneously to: (1) the lowest class of post-fire NIR reflectance and (2) to one of the two highest classes of NIR difference values. Of 323,259 pixels, 93,643 (circa 29\%) were selected as training samples for both PROVA-V tiles. Figure 3 shows the cumulative density functions for post-fire NIR reflectance values (Figure 3a) and NIR difference values (Figure 3b), and the thresholds for including pixels in the training sample.

(a)

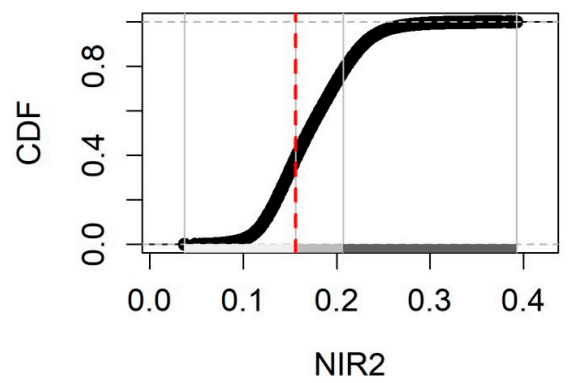

(b)

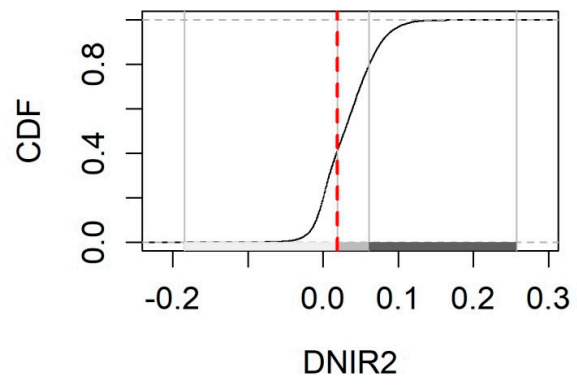

Figure 3. (a) Cumulative density function (CDF) and Jenks natural breaks for post-fire near-infrared reflectance (NIR) reflectance values (T2); (b) Cumulative density function (CDF) and Jenks natural breaks for pre-fire minus post-fire NIR reflectance differences. Red dashed lines represent thresholds for sample selection. Black arrows show values admitted for sample selection.

\subsubsection{Burned Area Classification-One-Class Support Vector Machine (OC-SVM) Classifier}

The training sample was then used as input to the One-class Support Vector Machine (OC-SVM) classifier. OC-SVM is a machine-learning algorithm derived from the standard Support Vector Machine algorithm [29] and designed to tackle single-class classification problems. It optimizes separation of the target class by constructing a hyperplane that best represents the multidimensional edge of the feature space. The hyperplane is obtained by minimizing the function:

$$
t(w, \xi, \rho)=\frac{1}{2}\|w\|^{2}-\rho+\frac{1}{m v} \sum_{i=1}^{m} \xi_{i}
$$

where $v$ is used to control the volume of the sphere that bounds the portion of the feature space where the training samples are located. It defines an upper limit to the outliers fraction found in the data [58], $w$ is the width of the region besides the decision boundary, and $m$ is the number of training instances.

Although developed for linear problems, different kernel functions for nonlinear problems can be used with OC-SVM [29]. Among the kernel functions available for computation, the most widely used are the radial basis function (RBF), the sigmoid function, and the polynomial function [50]. Kernel choice is based on the data type and on the $n$-dimensional feature distribution. In the present analysis we used the RBF kernel in the present analysis which has been extensively and successfully used in remote sensing image-processing tasks [59,60].

According to the boundaries defined by the kernel function of the OC-SVM, the classifier returns positive values for classes that are similar to the classes in the training sample and negative values otherwise. Computations were carried out using the R-Studio and the package e1071 [61]. The $v$ value is defined as the inverse of the number of features, which in the present study is equal to two (NIR values and NIR differences). The value of $w$ may vary between 0 , which yields low omission error at the expense of a higher comission, and 1 , which has the opposite effect. We selected this value as 0.1 , after a series of tests and visual analyses of the trials outputs. Finally, a morphological opening (erosion followed by dilation) filter by a square structuring element of size three was applied to the burned area map. 


\subsection{Accuracy Assessment}

The accuracy of AQM-PROBA-V burned area maps was assessed using reference fire perimeters extracted from each L8 scene and compared with the results from the MCD64A1 burned area product. We evaluated the AQM-PROBA-V and MCD64A1 in two different ways: (1) pixel-based and (2) grid-based. The pixel-based analysis was based on a contingency table (Table 2), from which different verification measures were calculated, namely overall accuracy $(\mathrm{OA})$, omission $(\mathrm{OE})$, and commission error (CE), bias (BIAS), Dice coefficients (DICE), and critical success index (CSI) (Table 3). The OA is the fraction of correctly classified pixels, either as burned or unburned. Accordingly, the OA reflects the agreement between the burned area (BA) product and the reference map (i.e., the accuracy of the classification) and satisfies the principle of equivalence of events, since it credits correct burned and unburned pixels equally [62]. This is not always a desirable attribute, particularly when the number of burned pixels is much smaller than the unburned one. An alternative to the OA is the CSI, which is useful when the event (burned pixels) occurs much less frequently than the nonevent (unburned pixels). It quantifies the proportion of correctly classified burned pixels, after removing correctly classified unburned pixels from consideration. The worst possible values for OA or CSI are zero, and the best is one. The $\mathrm{OE}$ and the $\mathrm{CE}$ provide respectively information about the reliability and discrimination power of the developed BA product, while the DICE is a measure of the overlap between the BA product and the reference map, in terms of the number of common burned pixels. The OE and CE range between zero and one, and have a reverse scale, such that smaller values are best. Conversely, the DICE has a direct scale, varying from zero (worst) to one (best). Finally, BIAS just indicates if the BA product overestimates (BIAS > 1) or underestimates (BIAS $<1$ ) the burned area, and should not be considered an accuracy measure, since it does not provide information about the correspondence between classification and reference. Unbiased BA products exhibit BIAS equal to 1, indicating that the burned event is classified the same number of times that it is observed.

Table 2. Generic contingency table between the reference and burned area (BA) products.

\begin{tabular}{ccccc}
\hline & & \multicolumn{3}{c}{ Reference } \\
\hline \multirow{4}{*}{ BA Products } & Burned & Burned & Unburned & Total \\
& Unburned & A & B & A + B \\
& Total & A $+C$ & D & C + D \\
& &
\end{tabular}

Table 3. Verification measures, acronyms and equations derived from contingency table from Table 2.

\begin{tabular}{ccc}
\hline Verification Measures & Acronym & Equation \\
\hline Overall Accuracy & OA & $(\mathrm{A}+\mathrm{D}) /(\mathrm{A}+\mathrm{B}+\mathrm{C}+\mathrm{D})$ \\
Omission Error & $\mathrm{OE}$ & $\mathrm{C} /(\mathrm{A}+\mathrm{C})$ \\
Commission Error & $\mathrm{CE}$ & $\mathrm{B} /(\mathrm{A}+\mathrm{C})$ \\
Bias & $\mathrm{BIAS}$ & $(\mathrm{A}+\mathrm{B}) /(\mathrm{A}+\mathrm{C})$ \\
Dice Coefficient & DICE & $2 \mathrm{~A} /(2 \mathrm{~A}+\mathrm{B}+\mathrm{C})$ \\
Critical Success Index & $\mathrm{CSI}$ & $\mathrm{A} /(\mathrm{A}+\mathrm{B}+\mathrm{C})$ \\
\hline
\end{tabular}

Most accuracy metrics available in the literature for the validation of thematic maps are derived from the contingency table. However, the traditional use of the contingency table assumes that reference data and classified data have the same spatial resolution, which is often not the case when evaluating coarse spatial resolution classifications, e.g., BA product acquired by sensors such as PROBA and MODIS. However, the influence of the sensor spatial resolution on the accuracy of the final burned area coarse product is well documented in the literature and is called low-resolution bias [49]. In order to circumvent this limitation, the contingency table was calculated using a fuzzy approach [63], which evaluates the proportion of reference $(30 \mathrm{~m})$ burned area contained within each pixel of the 
lower resolution burned area products ( $300 \mathrm{~m}$ and $500 \mathrm{~m}$, for PROBA-V and MODIS, respectively). This proportion was used to weigh the contingency table data. For example, if an AQM-PROBA burned pixel has $70 \%$ of its area considered as burned in the reference data, this pixel has a hit of 0.7 and a commission error of 0.3 . On the other hand, if the burned area in the reference data corresponds to $30 \%$ of an AQM-PROBA pixel, none of which is captured by AQM-PROBA, this pixel will have an omission error of 0.3 . The proportions were used as weights to calculate the contingency table, which results from the summation of all weighted values for each error or hit. The analysis takes into account the extent to which the lower resolution information diverges from the higher resolution reference. This approach has been used for validating burned area maps derived from low-spatial resolution data against reference data of higher spatial resolution, since it is more appropriate than the traditional contingency table for the comparison of datasets with different spatial resolutions [7,50,64]. Moreover, a confidence interval was calculated for each verification measure using AQM-PROBA and MDC64A1 burn maps, by means of the Z-test. The confidence interval means that by repeating the analysis with independent samples, the results will be within the upper and lower bounds of the interval with a pre-defined probability, in our case $95 \%$.

In the second approach, grid-based, we compared AQM-PROBA, MCD64A1, and the Landsat reference data over $10 \times 10 \mathrm{~km}$ grids for each L8 reference scene. The Kendall rank coefficient [65] was used to assess the correlation between the BA products within the $10 \times 10 \mathrm{~km}$ grid. The advantage of grid correlation analysis is that it quantifies regionally the overall agreement between burned area estimation and the reference, and has been widely used to study the relationship between burned area estimates at different spatial resolutions [15].

The Mann-Kendall test was used in order to check the null hypothesis (H0) of no correlation between reference maps and the developed maps $(\tau \leq 0)$. The alternative hypothesis (H1) indicates that there is a correlation between both reference and developed maps $(\tau>0)$, at a significance level of 0.05 .

Finally, we also evaluated classification errors according to the distribution of fire-scars size by number and by area for each Landsat scene. Thus, the reference, AQM-PROBA and MCD64A1 maps scars were categorized into four classes according to the fire-scar area: (1) very small (0-25 ha); (2) small (25-100 ha); (3) medium (100-1000 ha) and (4) large (>1000 ha).

\section{Results}

The AQM-PROBA algorithm performance was assessed through a comparison with the MCD64A1 product and the Landsat fire reference perimeters. For the whole study area, $12,848 \mathrm{~km}^{2}$ of the burned area were mapped using the AQM-PROBA algorithm, and 10,332 $\mathrm{km}^{2}$ for MCD64A1, against $13,086 \mathrm{~km}^{2}$ mapped in the reference map. In general, the overall accuracy for AQM-PROBA and MCD64A1 were high (Table 4), which is expected since the target class is much less frequent than the non-occurrence class.

Figure 4 shows boxplots for the accuracy measures of all the L8 scenes, for both BA products. AQM-PROBA yielded higher bias values than MCD64A1 (Figure 4c), whereas both products showed similar results for DICE and CSI (Figure 4d,e). AQM-PROBA showed lower mean values of OE $(30 \%)$ and higher CE (22\%) than the MCD64A1 (34\% and 15\% of OE and CE, respectively).

The verification measures for both products were also assessed individually for each Landsat scene (Table 4). The highest OE and CE values were recorded for the 221/071 scene, while BIAS values ranged from 0.40 (for MCD64A1 in the scene 221/071) to 1.18 (AQM-PROBA for 219/068). The DICE and CSI coefficients have similar values, with highest values for scenes 219/072, 220/68 and 222/67 (AQM-PROBA) and 219/068, 219/070, 220/067 (AQM-PROBA and MCD64A1). 


\section{官 AQM-PROBA 白 MCD64A1}

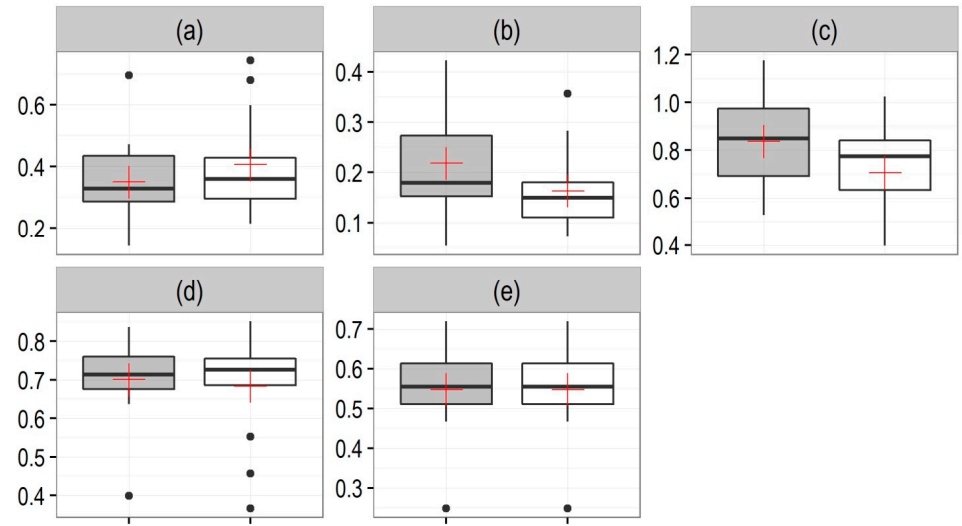

Figure 4. Boxplots for each verification measures (a) Omission Error (OE); (b) Comission Error (CE); (c) Bias (BIAS); (d) Dice Coefficient (DICE) and (e) Critical Success Index (CSI) for 13 Landsat 8 scenes based on Área Queimada-Project for On-Board Autonomy-Vegetation burned area product (AQM-PROBA) (gray boxes) and Moderate Resolution Imaging Spectroradiometer direct broadcast (DB) burned area product (MCD64A1) (white boxes), depicting the 25th, 50th (median, black line) and 75 th percentiles. The red cross represents the mean value, and black points are the outliers.

Table 4. Verification measures from contingency table (Omission Error (OE); Comission Error (CE); Bias (BIAS); Dice Coefficient (DICE) and Critical Success Index (CSI)) for Área Queimada-Project for On-Board Autonomy-Vegetation burned area product (AQM-PROBA) and Moderate Resolution Imaging Spectroradiometer direct broadcast (DB) burned area product (MCD64A1) for each Landsat 8 scene.

\begin{tabular}{cccccccc}
\hline Products & Path/Row & OA & OE & CE & BIAS & DICE & CSI \\
\hline AQM-PROBA & $218 / 072$ & 0.997 & 0.47 & 0.05 & 0.56 & 0.68 & 0.51 \\
MCD64A1 & $218 / 072$ & 0.996 & 0.68 & 0.21 & 0.41 & 0.46 & 0.30 \\
AQM-PROBA & $219 / 068$ & 0.990 & 0.14 & 0.27 & 1.18 & 0.79 & 0.65 \\
MCD64A1 & $219 / 068$ & 0.994 & 0.21 & 0.07 & 0.85 & 0.85 & 0.74 \\
AQM-PROBA & $219 / 070$ & 0.993 & 0.17 & 0.15 & 0.97 & 0.84 & 0.72 \\
MCD64A1 & $219 / 070$ & 0.993 & 0.22 & 0.11 & 0.88 & 0.83 & 0.71 \\
AQM-PROBA & $219 / 071$ & 0.998 & 0.43 & 0.27 & 0.78 & 0.64 & 0.47 \\
MCD64A1 & $219 / 071$ & 0.998 & 0.60 & 0.12 & 0.45 & 0.55 & 0.38 \\
AQM-PROBA & $219 / 072$ & 0.998 & 0.29 & 0.18 & 0.86 & 0.76 & 0.61 \\
MCD64A1 & $219 / 072$ & 0.998 & 0.40 & 0.07 & 0.65 & 0.73 & 0.57 \\
AQM-PROBA & $220 / 066$ & 0.986 & 0.31 & 0.32 & 1.03 & 0.69 & 0.52 \\
MCD64A1 & $220 / 066$ & 0.988 & 0.41 & 0.18 & 0.72 & 0.69 & 0.52 \\
AQM-PROBA & $220 / 067$ & 0.989 & 0.23 & 0.27 & 1.05 & 0.75 & 0.60 \\
MCD64A1 & $220 / 067$ & 0.991 & 0.30 & 0.16 & 0.84 & 0.76 & 0.62 \\
AQM-PROBA & $220 / 068$ & 0.996 & 0.29 & 0.16 & 0.85 & 0.77 & 0.63 \\
MCD64A1 & $220 / 068$ & 0.994 & 0.26 & 0.28 & 1.02 & 0.73 & 0.57 \\
AQM-PROBA & $221 / 067$ & 0.987 & 0.40 & 0.13 & 0.69 & 0.71 & 0.55 \\
MCD64A1 & $221 / 067$ & 0.988 & 0.34 & 0.15 & 0.78 & 0.74 & 0.59 \\
AQM-PROBA & $221 / 070$ & 0.991 & 0.35 & 0.31 & 0.95 & 0.67 & 0.50 \\
MCD64A1 & $221 / 070$ & 0.993 & 0.36 & 0.18 & 0.78 & 0.72 & 0.56 \\
AQM-PROBA & $221 / 071$ & 0.993 & 0.70 & 0.42 & 0.53 & 0.40 & 0.25 \\
MCD64A1 & $221 / 071$ & 0.994 & 0.74 & 0.36 & 0.40 & 0.37 & 0.22 \\
AQM-PROBA & $222 / 067$ & 0.989 & 0.33 & 0.17 & 0.81 & 0.74 & 0.59 \\
MCD64A1 & $222 / 067$ & 0.989 & 0.33 & 0.14 & 0.78 & 0.75 & 0.61 \\
AQM-PROBA & $222 / 068$ & 0.996 & 0.45 & 0.12 & 0.63 & 0.68 & 0.51 \\
MCD64A1 & $222 / 068$ & 0.996 & 0.43 & 0.10 & 0.63 & 0.70 & 0.54 \\
\hline
\end{tabular}


Rather than presenting just a single value for each verification measure, a confidence interval can be calculated and presented as part of the classification skill. The confidence interval is comprised by the range, which is the lower and upper limit of each metric that can be expected from the classification approach, and by the probability that the verification measures of the classification approach will fall within the range.

Accordingly, Table 5 indicates the expected range of each metric using a confidence interval of $95 \%$, which means that there is a likelihood of $95 \%$ that the confidence interval (lower-upper limits) encompasses the true classification metric on unseen data. The confidence interval results (Table 5) indicate that if the verification measure were calculated for different reference datasets, the average value of each metric would fall between the lower and upper limits of the interval, with high probability. Thus, the AQM-PROBA yielded better results for the OE, BIAS, DC, CSI verification measures than MCD64A1, which, in turn, has a lower CE.

Table 5. Confidence intervals calculated with the Z-test with $95 \%$ confidence, for each verification measures (Omission Error (OE); Comission Error (CE); Bias (BIAS); Dice Coefficient (DICE) and Critical Success Index (CSI)) from contingency table and for each burned area product (Área Queimada-Project for On-Board Autonomy-Vegetation burned area product (AQM-PROBA) and Moderate Resolution Imaging Spectroradiometer direct broadcast (DB) burned area product (MCD64A1)).

\begin{tabular}{cccc}
\hline Verification Measures & Lower Limit & Upper Limit & Fire Product \\
\hline \multirow{2}{*}{ OE } & 0.27 & 0.42 & AQM-PROBA \\
& 0.31 & 0.5 & MCD64A1 \\
\hline \multirow{2}{*}{ CE } & 0.16 & 0.27 & AQM-PROBA \\
& 0.11 & 0.2 & MCD64A1 \\
\hline \multirow{2}{*}{ BIAS } & 0.73 & 0.94 & AQM-PROBA \\
& 0.6 & 0.81 & MCD64A1 \\
\hline \multirow{2}{*}{ DC } & 0.64 & 0.75 & AQM-PROBA \\
& 0.6 & 0.76 & MCD64A1 \\
\hline \multirow{2}{*}{ CSI } & 0.48 & 0.61 & AQM-PROBA \\
& 0.45 & 0.61 & MCD64A1 \\
\hline
\end{tabular}

Comparing the total burned area obtained from reference data, AQM-PROBA and MCD64A1 for each Landsat scene it is clear that AQM-PROBA displayed results closer to the reference data than MCD64A1, with the exception of the 220/68, 221/67, 222/67 and 222/68 scenes. Figure 5 shows different fire patterns among the thirteen analyzed scenes. Three scenes $(218 / 072,219 / 071$ and 219/072) present less than $400 \mathrm{~km}^{2}$ of burned area, whereas another three scenes contain approximately $600-750 \mathrm{~km}^{2}$ of burned area $(220 / 068,222 / 068$, and 221/071). Finally, the majority (seven scenes, 219/068, 219/070, 220/066, 220/067, 221/067, 221/070 and 222/067) display more than $1200 \mathrm{~km}^{2}$ of area burned each. While all three BA products show similar spatial behavior, there are significant differences regarding the magnitude of the amount of burned area. On average, the AQM-PROBA and MCD64A1 provide underestimation of BA compared to the reference Landsat. The AQM-PROBA (MCD64A1) product provides greater burned area amount in 8 (5) of the analyzed scenes than MCD64A1 (AQM-PROBA), corroborating the BIAS behavior shown in Table 4.

A better insight into the fire pattern of each analyzed scene may be obtained by analyzing the distribution of fire scars size by the corresponding fractions of total number of scars and of total burned area. Accordingly, Figure 6 shows the reference fire scar size distribution by number of scar categorized into four classes: (1) very small (0-25 ha); (2) small (25-100 ha); (3) medium (100-1000 ha); and (4) large (>1000 ha). Figure 6 reveals that the region presents a predominance of very small fire scars (class 1), however these scars accounts for less than $5 \%$ of the total amount of burned area. Conversely, the highest amount of burned area belongs to large fire scars (class 4, larger than $1000 \mathrm{ha}$ ), which in turn, accounts for the lowest number of scars. 


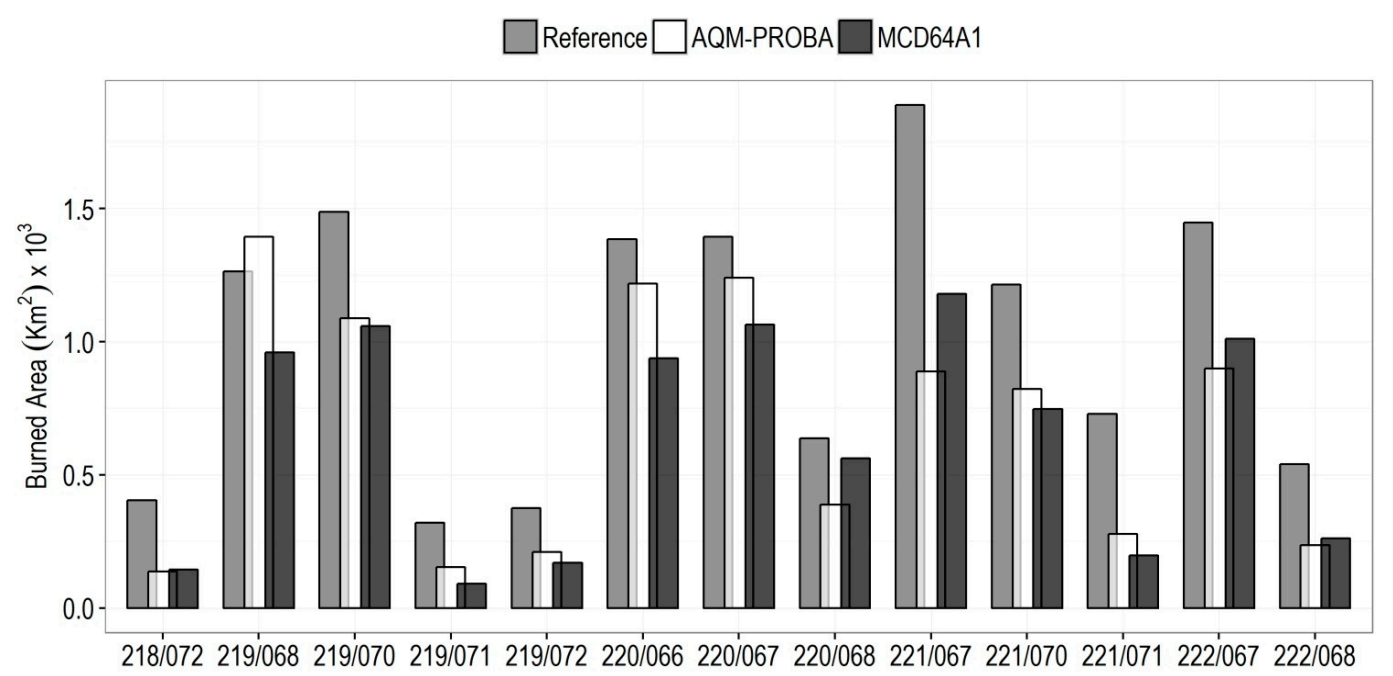

Figure 5. Total burned area for the 13 Landsat scenes, as obtained from the reference data (gray bars), Área Queimada-Project for On-Board Autonomy-Vegetation burned area product (AQM-PROBA) (white bars) and Moderate Resolution Imaging Spectroradiometer direct broadcast (DB) burned area product (MCD64A1) (black bars).

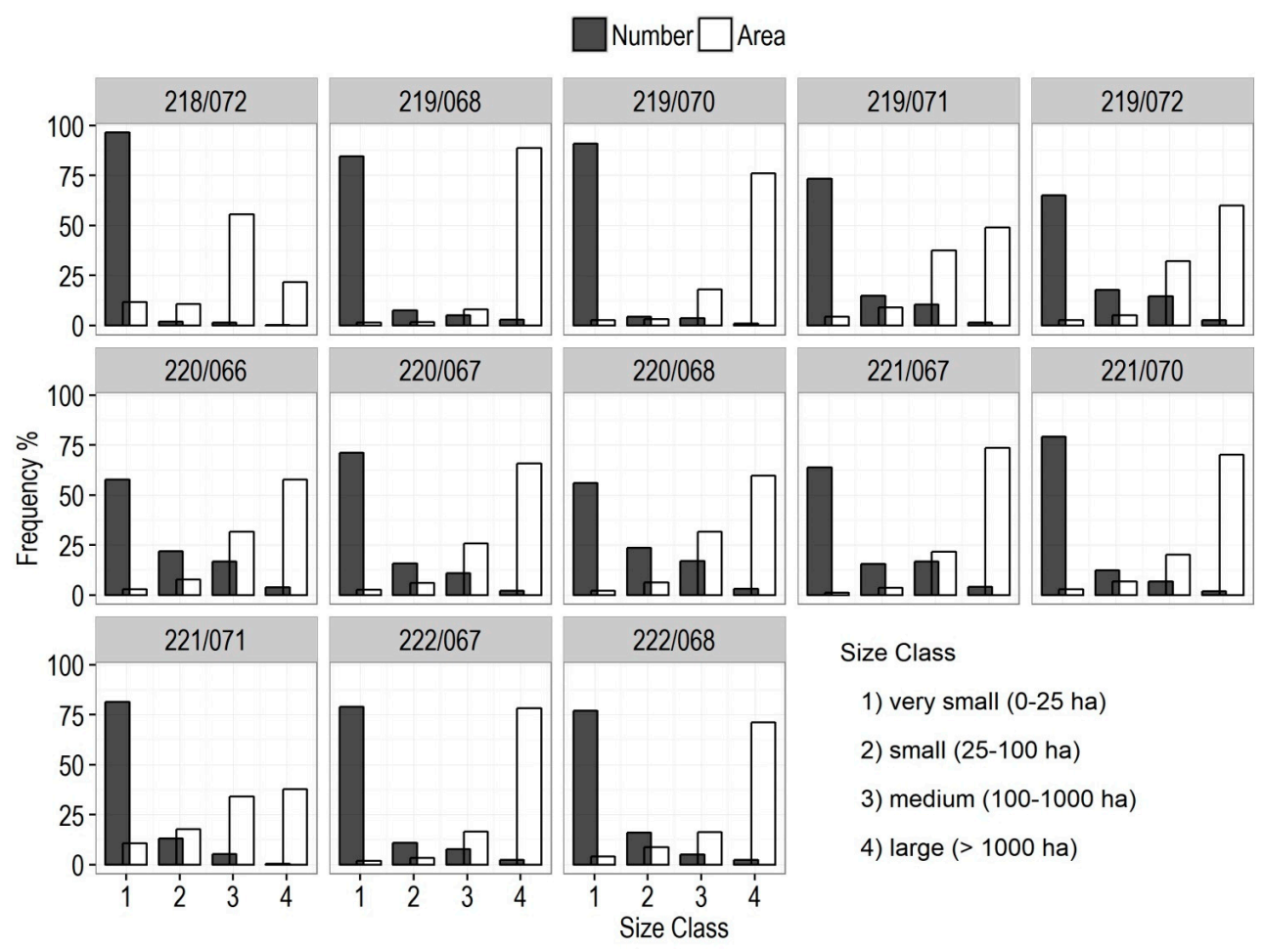

Figure 6. Distribution of reference fire scars by number (gray bars) and by area (white bars) according to each size class (1) very small (0-25 ha); (2) small (25-100 ha); (3) medium (100-1000 ha) and (4) large (>1000 ha), for each Landsat 8 scene.

The L8 scenes 219/71 and 221/71 showed smaller percent differences in between size classes 100-1000ha and $>1000$ ha (Figure 6), in comparison to other scenes. These scenes also displayed high omission error for both products (Table 4). Nevertheless, Figure 7 shows that most omissions cases are due to small fire scars (AQM-PROBA in red, MCD64A1 in blue and, black contour represents the reference data). 

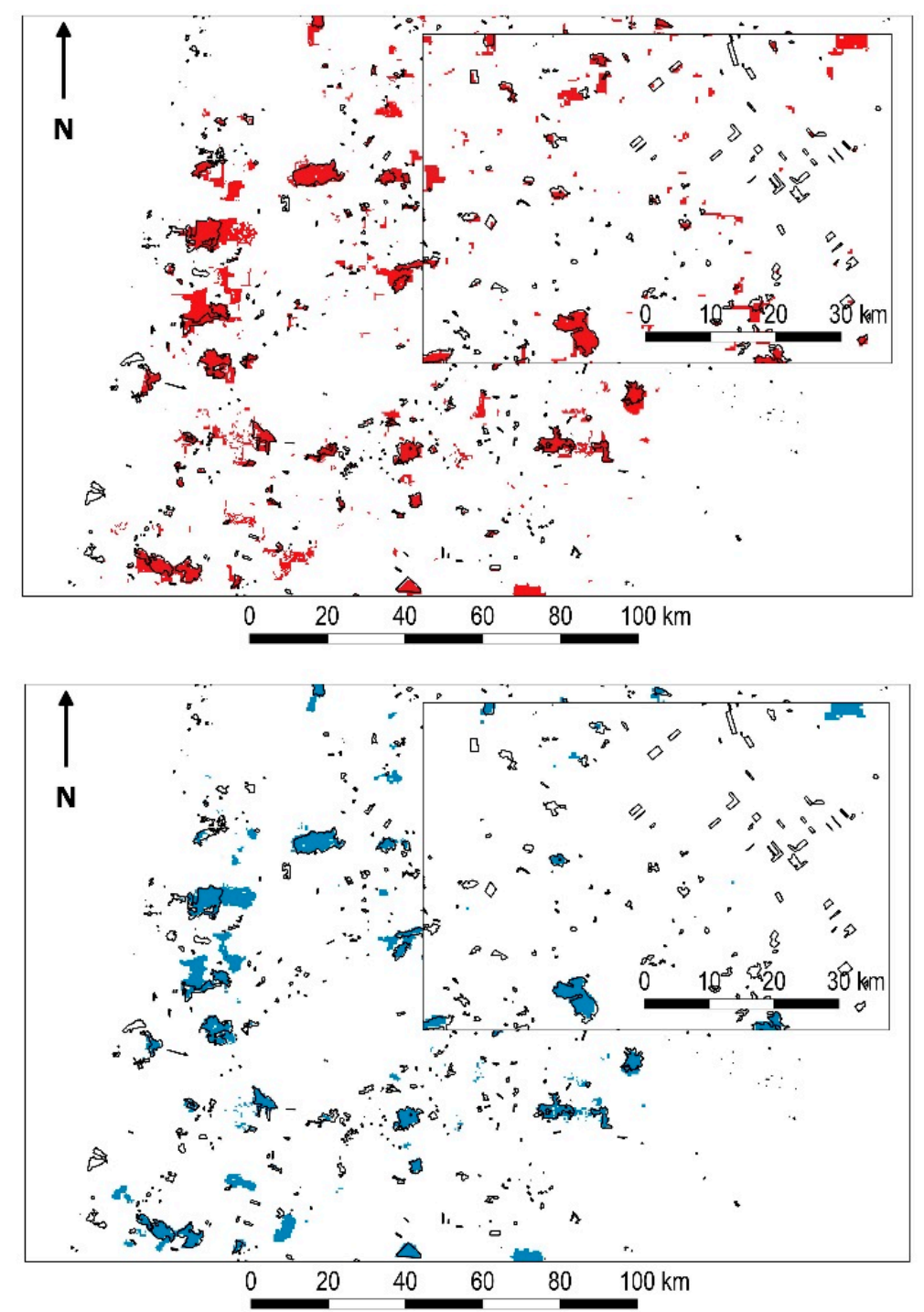

Figure 7. Burned area maps for Área Queimada-Project for On-Board Autonomy-Vegetation burned area product (AQM-PROBA) (red) and Moderate Resolution Imaging Spectroradiometer direct broadcast (DB) burned area product (MCD64A1) (blue) for 219/071 scene. The black outline represents the reference Landsat 8 fire scars perimeters. The small rectangles highlight examples of omissions cases related to small fire scars.

Figure 8 shows the burned area proportions classified in all the Landsat scenes plotted against the proportion classified as burned in the two BA products in a region of 10 by $10 \mathrm{~km}$ defined within each reference data scene. For all scenes, the AQM-PROBA showed a greater correlation with the Landsat burned area estimates than the MCD64A1 product. Both BA products underestimate the BA in most cases, except in 219/68, 219/70, 222/67 for AQM-PROBA-V and 220/68 for MCD64A1 (Figure 8).

Table 6 shows the Kendall correlation index calculated for each product and for each Landsat scene. For AQM-PROBA, the highest Kendall's correlation was found for scene 220/66 (0.85) and the lowest for scene 218/72 (0.51). For MCD64A1, the best result was found for scene 221/67 (0.72) and the worst for scene 218/72 (0.37). On average, the correlation between AQM-PROBA and the reference map was 0.7 , with a standard deviation of 0.09 , whereas for MCD64A1 the correlation was 0.56 with a standard deviation of 0.11 (Table 6). 
Path/row - 218/072
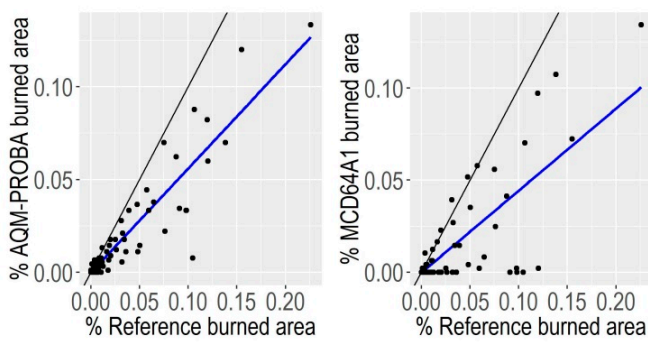

(a)

Path/row - 219/070
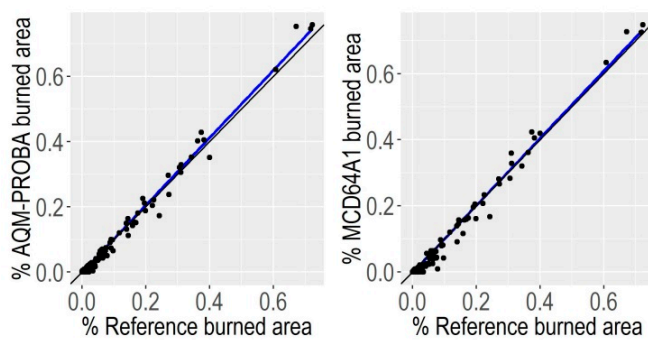

(c)

Path/row - 219/072
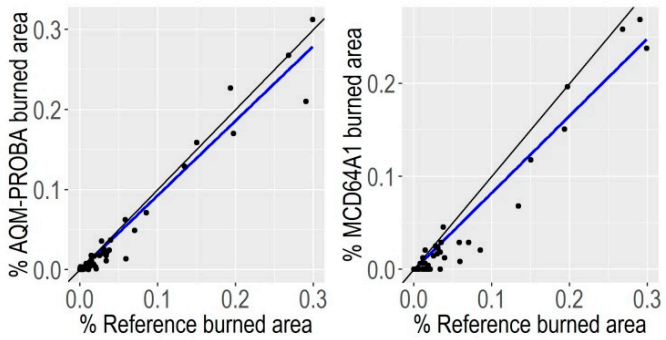

(e)

Path/row - 220/067
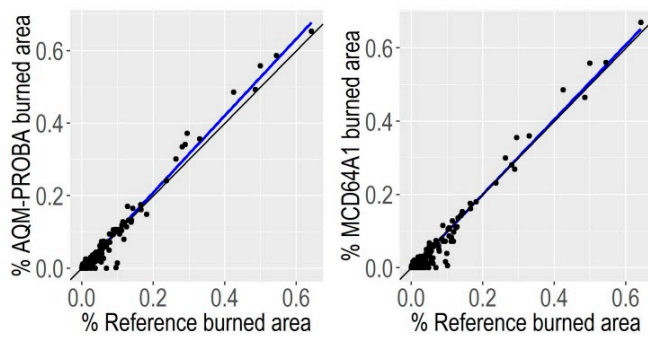

(g)

Path/row - 221/067
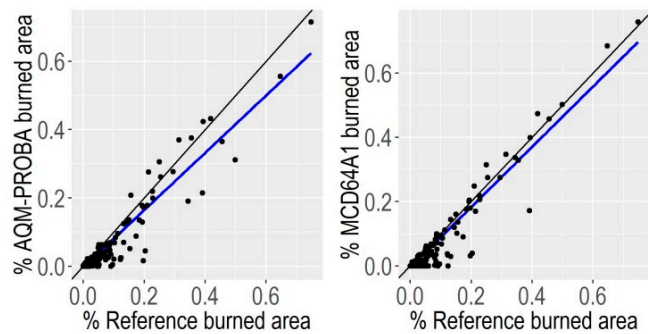

(i)
Path/row - 219/068
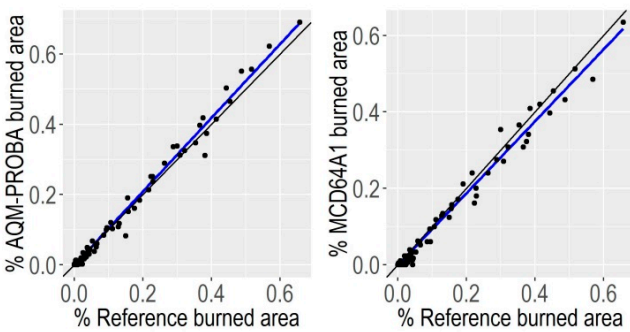

(b)

Path/row - 219/071

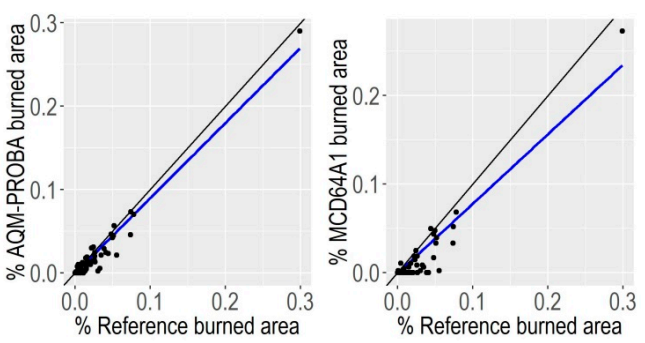

(d)

Path/row - 220/066

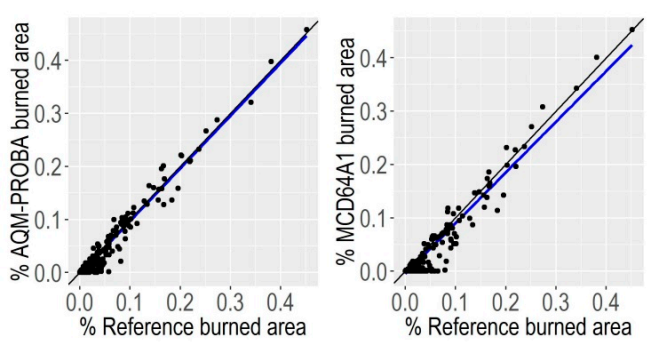

(f)

Path/row - 220/068

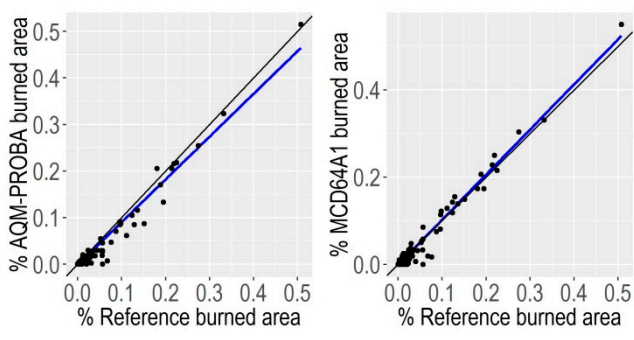

(h)

Path/row - 221/070
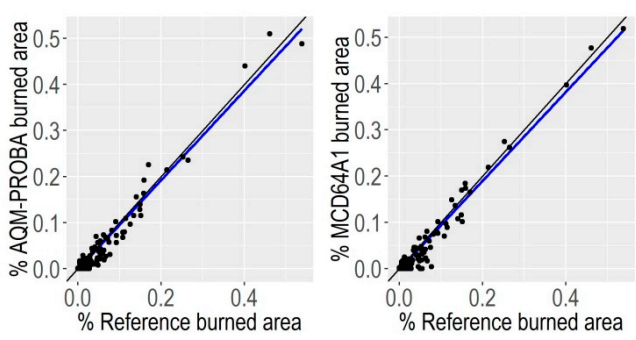

(j)

Figure 8. Cont. 
Path/row - 221/071
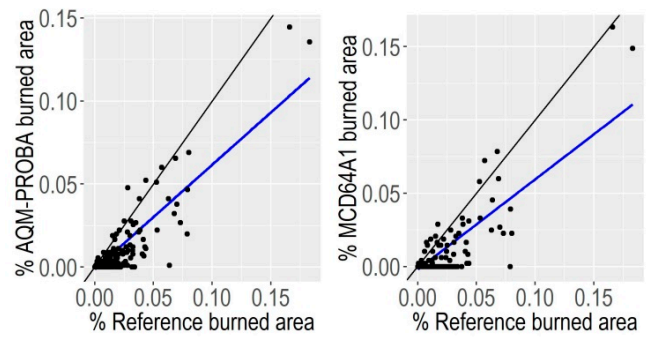

$(\mathbf{k})$

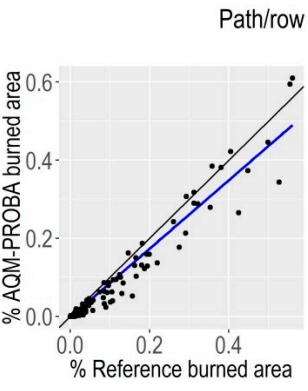

Path/row - 222/067

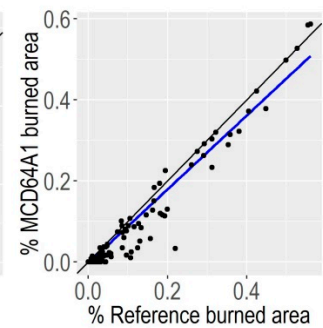

(1)

Path/row - 222/068
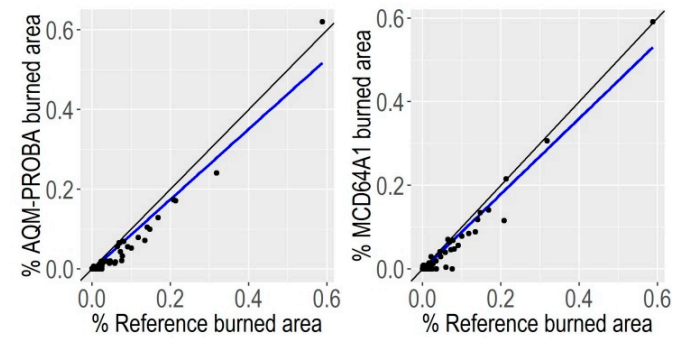

$(\mathbf{m})$

Figure 8. Scatterplots between burned area classified with the Área Queimada-Project for On-Board Autonomy-Vegetation burned area product (AQM-PROBA) and Moderate Resolution Imaging Spectroradiometer direct broadcast (DB) burned area product (MCD64A1) algorithms and the reference fire perimeters for a defined $10 \times 10 \mathrm{~km}$ grid within each Landsat 8 scene represented by each subfigure (8a to 81 ). The regression line (blue) and the 1:1 line (black) are also displayed.

Table 6. Values obtained from the calculation of the Kendall correlation index $(\tau)$ with a confidence level of 95\% for both products Área Queimada-Project for On-Board Autonomy-Vegetation burned area product (AQM-PROBA) and and Moderate Resolution Imaging Spectroradiometer direct broadcast (DB) burned area product (MCD64A1) and for each Landsat 8 scene (path/row).

\begin{tabular}{ccc}
\hline Product & Path/Row & $\boldsymbol{\tau}$ \\
\hline AQM-PROBA & $218 / 072$ & 0.51 \\
MCD64A1 & $218 / 072$ & 0.37 \\
AQM-PROBA & $219 / 068$ & 0.72 \\
MCD64A1 & $219 / 068$ & 0.60 \\
AQM-PROBA & $219 / 070$ & 0.69 \\
MCD64A1 & $219 / 070$ & 0.61 \\
AQM-PROBA & $219 / 071$ & 0.66 \\
MCD64A1 & $219 / 071$ & 0.43 \\
AQM-PROBA & $219 / 072$ & 0.71 \\
MCD64A1 & $219 / 072$ & 0.53 \\
AQM-PROBA & $220 / 066$ & 0.85 \\
MCD64A1 & $220 / 066$ & 0.65 \\
AQM-PROBA & $220 / 067$ & 0.79 \\
MCD64A1 & $220 / 067$ & 0.68 \\
AQM-PROBA & $220 / 068$ & 0.76 \\
MCD64A1 & $220 / 068$ & 0.67 \\
AQM-PROBA & $221 / 067$ & 0.82 \\
MCD64A1 & $221 / 067$ & 0.72 \\
AQM-PROBA & $221 / 070$ & 0.70 \\
MCD64A1 & $221 / 070$ & 0.63 \\
AQM-PROBA & $221 / 071$ & 0.67 \\
MCD64A1 & $221 / 071$ & 0.42 \\
AQM-PROBA & $222 / 067$ & 0.72 \\
MCD64A1 & $222 / 067$ & 0.60 \\
AQM-PROBA & $222 / 068$ & 0.58 \\
MCD64A1 & $222 / 068$ & 0.44 \\
\hline
\end{tabular}




\section{Discussion}

Two aspects can be considered in the analysis of omission and commission errors: the first is related to errors along the edges of burn scars, due to the differences in spatial resolution of the datasets, possibly combined with georeferencing errors. The second is related to fires that occurred prior to the date of the first Landsat image, but were detected in the PROBA-V composite after this date, probably due to shortage of cloud-free data. This type of error was also identified in the results of MCD64A1. The uncertainty in detection dates can lead to this type of error in the accuracy assessment [52].

Although the AQM-PROBA and MCD64A1 products are based on different data derived from different sensors, the divergences in the results presented may reflect the different methodologies applied. Both products use active fire for spectral characterization of burned pixels, but they are different in design. The AQM-PROBA is based on the spectral space of the NIR T2 and NIR T1-T2 values in PROBA-V images, with samples collected by VIIRS active fire, which is used to train the OC-SVM classification model. On the other hand, MCD64A1 uses spectral index data constructed in the NIR-SWIR spectral space $(1.6$ and $2.1 \mu \mathrm{m})$ and cumulative active fire maps to guide the selection of burned and unburned samples and to specify probabilities. In addition, the better spatial resolution of PROBA-V compared to MODIS data and the use of OC-SVDD may have an influence on the detection of smaller fire scars. In the AQM-PROBA product, isolated pixels are excluded from the final result, such that the minimum mapping area is $22 \mathrm{ha}$. Due to the $500 \mathrm{~m}$ spatial resolution of MODIS, the theoretical minimum area mapped by the MCD64A1 would be 50 ha. However, according to previous studies, it is hard to detect burned areas smaller than about 4 to 10 pixels in size [64]. For instance, the minimum resolution at which MCD64A1 is reliable is 120 ha [66].

Both products showed the same trend towards larger omission errors and lower correlation with the reference data when small burns dominate the fire size distribution. This is common when comparing images and reference data collected at different spatial resolutions, where the size and irregularity of the targets are determinant for higher omission errors [5,7,15,52]. The largest omission errors in both burned area products were observed where scars are typically small. Silva et al. [15] using Satellite Pour l'Observation de la Terre (SPOT) Vegetation data over Africa, mentioned two atypical situations: small burned areas that were sufficiently dark to be detected (low values of NIR reflectance after the fire) and large burned areas that were frequently missed due to a small decrease in NIR reflectance values after the fire. The former case may explain the results obtained with scene 219/072 evaluated in our study. This scene contained small burned areas but yielded a Kendall coefficient of 0.71 for AQM-PROBA, similar to those obtained for areas with larger burns. Besides the presence of small burned areas, scene $218 / 72$ covers a peculiar area where a rugged topography and extensive rock outcrops further complicated burned area detection with low-spatial resolution sensors.

Thus, we show that mapping accuracy is likely to vary significantly in space due to the observed contrast between the distribution of fire size number and area. The extent and fragmentation pattern of burning, namely on the relative importance of small versus large burns, introduces limitations in BA estimation when using coarse spatial resolution sensors. Since the study area is characterized by a high frequency of very small scars ( $<25 \mathrm{ha})$, a large number of omission errors was observed due to the coarse resolution of the BA products (500 $\mathrm{m}$ for MCD64A1 and $350 \mathrm{~m}$ for AQM-PROBA), in some cases leading to a substantial underestimation of total area burned. On the order hand, in regions with predominance of large fire scars, the BA coarse products are quite accurate, and may even lead to slight overestimation. High reflectance values of NIR after the fire may be the reason for the results of scene 221/067, where omission errors were high, despite the occurrence of large burns. One possible cause is the presence of low fuel loadings in the area, and the presence of quartz neosols [67]. This soil has a whitish color and high reflectance values of the NIR channel due to the presence of sand and quartz in their composition [68]. Libonati [7] validated the AQM algorithm for scene 221/067 considering a multi temporal data set spanning a six-year period. This algorithm uses the index $W$, constructed by the spectral space of the NIR-MIR (middle infrared) spectral region of the MODIS sensor. The AQM algorithm is based on the MODIS MIR channels, suggesting that the use of different channels and 
indices may produce better results in the area. In this case, the spectral space of the MIR, used in AQM, can have a positive influence.

\section{Conclusions}

This study highlights the use of a One-Class Support Vector Machine classifier for burned area mapping using automated sample selection based on active fires. The procedure was applied to the Brazilian Savanna using Project for On-Board Autonomy-Vegetation (PROBA-V) reflectance and Visible Infrared Imaging Radiometer Suite (VIIRS) active fire data. Nevertheless, we must highlight that although the present study has used PROBA-V images and VIIRS active fire data, the approach may be easily adapted to other data sources.

Validation results using Landsat reference data, and a comparison with the MCD64A1 burned area product (MODIS/Terra and Aqua Burned Area Monthly L3 Global $500 \mathrm{~m}$ ) showed a higher accuracy of the current algorithm in the Brazilian Cerrado. In general, the AQM-PROBA presented lower omission error compared with the MCD64A1 product, but the latter displayed lower commission errors. However, the AQM-PROBA displayed more balanced values of omission and commission and, consequently, a bias closer to 1 and a higher correlation with the fire reference data. Higher similarity was also observed between reference and AQM-PROBA for fire scars smaller than 100 ha.

One of the limitations of the presented methodology is the spectral limitation of the PROBA-V sensor, which has only four channels. Several studies have shown that the spectral space of short wave infra-red and middle infra-red channels in savanna areas is advantageous, especially at the 2.1 and $3.9 \mu \mathrm{m}$ wavelengths, respectively [69-73]. Thus, the implementation of the approach presented herein with spectral indexes presenting greater separability between burned and unburned surfaces may improve the results. Considering that burned areas in the Cerrado tend to show an increase in temperature after the fire occurrence [74], another alternative to improve burned area detection may involve the use of thermal data.

Finally, the proposed methodology based on the use of one-class algorithms opens new perspectives on fire scar detection, since current burned area algorithms based on hybrid approaches are still hampered by the accuracy of active fire products. Moreover, the results of this study have demonstrated that the technique of automated sample selection based on active fires as proposed here produced accurate and promising results for burned area mapping, circumventing the need for human intervention in sample acquisition.

Acknowledgments: The authors acknowledge the support of the Science without Borders Program from CNPq 407459/2013-8, grants 215158/2014-8 and 150625/2017-0; the FAPESP/FCT Project Brazilian Fire-Land-Atmosphere System (BrFLAS) 1389/2014 and 2015/01389-4; and the scientific grant FAPERJ (E-26/203.174/2016). The first author also acknowledges CAPES for the grants to a Doctoral program of Teacher Training of IFSULDEMINAS. The Centro de Estudos Florestais (CEF) is a research unit funded by Fundação para a Ciência e Tecnologia I.P. (FCT), Portugal (UID/AGR/00239/2013).

Author Contributions: Allan A. Pereira and José Miguel C. Pereira developed the AQM-PROBA algorithm and performed the validation approach. Renata Libonati established the methodology for the validation section and compiled the manuscript. Alberto W. Setzer, Duarte Oom, Fabiano Morelli, Luis M. T. Carvalho and Fausto Machado-Silva reviewed the paper and contributed to the interpretation of the results.

Conflicts of Interest: The authors declare no conflict of interest.

\section{References}

1. Bowman, D.M.J.S.; Balch, J.K.; Artaxo, P.; Bond, W.J.; Carlson, J.M.; Cochrane, M.A.; D'Antonio, C.M.; DeFries, R.S.; Doyle, J.C.; Harrison, S.P.; et al. Fire in the Earth System. Science 2009, 324, 481-484. [CrossRef] [PubMed]

2. FAO. Forest and Other Vegetation Fires. Available online: http://www.fao.org/forestry/firemanagement/ en/ (accessed on 23 October 2017). 
3. Brannstrom, C.; Jepson, W.; Filippi, A.M.; Redo, D.; Xu, Z.; Ganesh, S. Land change in the Brazilian Savanna (Cerrado), 1986-2002: Comparative analysis and implications for land-use policy. Land Use Policy 2008, 25, 579-595. [CrossRef]

4. Fidelis, A.; di Santi Lyra, M.F.; Pivello, V.R. Above- and below-ground biomass and carbon dynamics in Brazilian Cerrado wet grasslands. J. Veg. Sci. 2013, 24, 356-364. [CrossRef]

5. Alonso-Canas, I.; Chuvieco, E. Global burned area mapping from ENVISAT-MERIS and MODIS active fire data. Remote Sens. Environ. 2015, 163, 140-152. [CrossRef]

6. Barbosa, P.; Barbosa, P.M.; Gre, J.; Pereira, M.C. An Algorithm for Extracting Burned Areas from Time Series of AVHRR GAC Data Applied at a Continental Scale An Algorithm for Extracting Burned Areas from Time Series of AVHRR GAC Data Applied at a Continental Scale. Remote Sens. Environ. 1999, 69. [CrossRef]

7. Libonati, R.; DaCamara, C.; Setzer, A.; Morelli, F.; Melchiori, A. An Algorithm for Burned Area Detection in the Brazilian Cerrado Using $4 \mu \mathrm{m}$ MODIS Imagery. Remote Sens. 2015, 7, 15782-15803. [CrossRef]

8. Simon, M.; Plummer, S.; Fierens, F.; Hoelzemann, J.J.; Arino, O. Burnt area detection at global scale using ATSR-2: The GLOBSCAR products and their qualification. J. Geophys. Res. D Atmos. 2004, 109. [CrossRef]

9. Quintano, C.; Fernández-Manso, A.; Stein, A.; Bijker, W. Estimation of area burned by forest fires in Mediterranean countries: A remote sensing data mining perspective. For. Ecol. Manag. 2011, 262, 1597-1607. [CrossRef]

10. Roy, D.P.; Jin, Y.; Lewis, P.E.; Justice, C.O. Prototyping a global algorithm for systematic fire-affected area mapping using MODIS time series data. Remote Sens. Environ. 2005, 97, 137-162. [CrossRef]

11. Giglio, L.; Loboda, T.; Roy, D.P.; Quayle, B.; Justice, C.O. An active-fire based burned area mapping algorithm for the MODIS sensor. Remote Sens. Environ. 2009, 113, 408-420. [CrossRef]

12. Pereira, J.M.C.; Sá, A.C.L.; Sousa, A.M.O.; Silva, J.M.N.; Santos, T.N.; Carreiras, J.M.B. Spectral characterisation and discrimination of burnt areas. In Remote Sensing of Large Wildfires; Springer: Berlin, Germany, 1999; pp. 123-138.

13. Mouillot, F.; Schultz, M.G.; Yue, C.; Cadule, P.; Tansey, K.; Ciais, P.; Chuvieco, E. Ten years of global burned area products from spaceborne remote sensing-A review: Analysis of user needs and recommendations for future developments. Int. J. Appl. Earth Obs. Geoinf. 2014, 26, 64-79. [CrossRef]

14. Hoelzemann, J.J.; Schultz, M.G.; Brasseur, G.P.; Granier, C.; Simon, M. Global Wildland Fire Emission Model (GWEM): Evaluating the use of global area burnt satellite data. J. Geophys. Res. D Atmos. 2004, 109. [CrossRef]

15. Silva, J.M.N.; Sá, A.C.L.; Pereira, J.M.C. Comparison of burned area estimates derived from SPOT-VEGETATION and Landsat ETM+ data in Africa: Influence of spatial pattern and vegetation type. Remote Sens. Environ. 2005, 96, 188-201. [CrossRef]

16. Nogueira, J.; Ruffault, J.; Chuvieco, E.; Mouillot, F. Can We Go Beyond Burned Area in the Assessment of Global Remote Sensing Products with Fire Patch Metrics? Remote Sens. 2016, 9, 7. [CrossRef]

17. Chuvieco, E.; Yue, C.; Heil, A.; Mouillot, F.; Alonso-Canas, I.; Padilla, M.; Pereira, J.M.; Oom, D.; Tansey, K. A new global burned area product for climate assessment of fire impacts. Glob. Ecol. Biogeogr. 2016, 25, 619-629. [CrossRef]

18. Roy, D.P. Multi-temporal active-fire based burn scar detection algorithm. Int. J. Remote Sens. 1999, 20, 1031-1038. [CrossRef]

19. Loboda, T.; O'neal, K.J.; Csiszar, I. Regionally adaptable dNBR-based algorithm for burned area mapping from MODIS data. Remote Sens. Environ. 2007, 109, 429-442. [CrossRef]

20. Roy, D.P.; Frost, P.G.H.; Justice, C.O.; Landmann, T.; Le Roux, J.L.; Gumbo, K.; Makungwa, S.; Dunham, K.; DU Toit, R.; Mhwandagaraii, K.; et al. The Southern Africa Fire Network (SAFNet) regional burned-area product-validation protocol. Int. J. Remote Sens. 2005, 26, 4265-4292. [CrossRef]

21. Schroeder, W.; Csiszar, I.; Morisette, J. Quantifying the impact of cloud obscuration on remote sensing of active fires in the Brazilian Amazon. Remote Sens. Environ. 2008, 112, 456-470. [CrossRef]

22. Justice, C.O.; Korontzi, S. A review of the status of satellite fire monitoring and the requirements for global environmental change research. In Global and Regional Vegetation Fire Monitoring from Space: Planning a Coordinated International Effort; SPB Academic Publishing: New York, NY, USA, 2001; p. 302.

23. Asner, G.P. Cloud cover in Landsat observations of the Brazilian Amazon. Int. J. Remote Sens. 2001, 22, 3855-3862. [CrossRef] 
24. Hilker, T.; Lyapustin, A.I.; Hall, F.G.; Myneni, R.; Knyazikhin, Y.; Wang, Y.; Tucker, C.J.; Sellers, P.J. On the measurability of change in Amazon vegetation from MODIS. Remote Sens. Environ. 2015, 166, $233-242$. [CrossRef]

25. Wylie, D.; Jackson, D.L.; Menzel, W.P.; Bates, J.J. Trends in global cloud cover in two decades of HIRS observations. J. Clim. 2005, 18, 3021-3031. [CrossRef]

26. Pimentel, M.A.F.; Clifton, D.A.; Clifton, L.; Tarassenko, L. A review of novelty detection. Signal Process. 2014, 99, 215-249. [CrossRef]

27. Mazher, A.; Li, P.; Zhang, J. Mapping burned areas from Landsat TM imags: A comparative study. In Proceedings of the IEEE International Conference on Computer Vision in Remote Sensing, Xiamen, China, 2012; pp. 285-290.

28. Song, B.; Li, P.; Li, J.; Plaza, A. One-Class Classification of Remote Sensing Images Using Kernel Sparse Representation. IEEE J. Sel. Top. Appl. Earth Obs. Remote Sens. 2016, 9, 1613-1623. [CrossRef]

29. Scholkopf, B.; Williamson, R.; Smola, A.; Shawe-Taylor, J.; Platt, J. Support Vector Method for Novelty Detection. NIPS 2000, 12, 582-588.

30. Myers, N.; Mittermeier, R.A.; Mittermeier, C.G.; da Fonseca, G.A.B.; Kent, J. Biodiversity hotspots for conservation priorities. Nature 2000, 403, 853-858. [CrossRef] [PubMed]

31. Da Silva, J.M.C.; Bates, J.M. Biogeographic Patterns and Conservation in the South American Cerrado: A Tropical Savanna Hotspot The Cerrado, which includes both forest and savanna habitats, is the second largest South American biome, and among the most threatened on the continent. Bioscience 2002, 52, $225-234$.

32. Hardesty, J.; Myers, R.; Fulks, W. Fire, ecosystems, and people: A preliminary assessment of fire as a global conservation issue. George Wright Forum 2005, 22, 78-87.

33. Pivello, V.R. The Use of Fire in the Cerrado and Amazonian Rainforests of Brazil: Past and Present. Fire Ecol. 2011, 7, 24-39. [CrossRef]

34. Durigan, G.; Ratter, J.A. The need for a consistent fire policy for Cerrado conservation. J. Appl. Ecol. 2016, 53, 11-15. [CrossRef]

35. Frizzo, T.L.M.; Bonizário, C.; Borges, M.P.; Vasconcelos, H.L. Revisão dos efeitos do fogo sobre a fauna de formações savânicas do Brasil. Oecol. Aust. 2011, 15, 365-379. [CrossRef]

36. Fiedler, N.C.; de Azevedo, I.N.C.; Rezende, A.V.; de Medeiros, M.B.; Venturoili, F. Effect of fire on the structure and floristic composition of a cerrado sensu stricto area in fazenda Água Limpa-DF. Rev. Árvore 2004, 28, 129-138. [CrossRef]

37. Ferreira, A.J.D.; Coelho, C.O.A.; Ritsema, C.J.; Boulet, A.K.; Keizer, J.J. Soil and water degradation processes in burned areas: Lessons learned from a nested approach. Catena 2008, 74, 273-285. [CrossRef]

38. Oliveira, P.T.S.; Nearing, M.A.; Moran, M.S.; Goodrich, D.C.; Wendland, E.; Gupta, H.V. Trends in water balance components across the Brazilian Cerrado. Water Resour. Res. 2014, 50, 7100-7114. [CrossRef]

39. Venter, O.; Sanderson, E.W.; Magrach, A.; Allan, J.R.; Beher, J.; Jones, K.R.; Possingham, H.P.; Laurance, W.F.; Wood, P.; Fekete, B.M.; et al. Sixteen years of change in the global terrestrial human footprint and implications for biodiversity conservation. Nat. Commun. 2016, 7, 12558. [CrossRef] [PubMed]

40. Silva, P.; Bastos, A.; DaCamara, C.C.; Libonati, R. Future projections of fire occurrence in Brazil using EC-Earth climate model. Rev. Bras. Meteorol. 2016, 31. [CrossRef]

41. Köppen, W.; Volken, E.; Brönnimann, S. The thermal zones of the Earth according to the duration of hot, moderate and cold periods and to the impact of heat on the organic world. Meteorol. Z. 2011, 20, 351-360. [CrossRef]

42. Libonati, R.; Dacamara, C.C. Spatial and temporal patterns of burned area over Brazilian Cerrado from 2005 to 2015 using remote sensing data. In Proceedings of the EGU General Assembly, Vienna, Austria, 17-22 April 2016.

43. Moreira de Araújo, F.; Ferreira, L.G.; Arantes, A.E. Distribution patterns of burned areas in the Brazilian biomes: An analysis based on satellite data for the 2002-2010 period. Remote Sens. 2012, 4, 1929-1946. [CrossRef]

44. Francois, M.; Santandrea, S.; Mellab, K.; Vrancken, D.; Versluys, J. The PROBA-V mission: The space segment. Int. J. Remote Sens. 2014, 35, 2548-2564. [CrossRef]

45. Sterckx, S.; Benhadj, I.; Duhoux, G.; Livens, S.; Dierckx, W.; Goor, E.; Adriaensen, S.; Heyns, W.; Van Hoof, K.; Strackx, G.; et al. The PROBA-V mission: Image processing and calibration. Int. J. Remote Sens. 2014, 35, 2565-2588. [CrossRef] 
46. VITO. Available online: http://ww1.vito.eodata.be/PDF/portal/Application.html\#Home (accessed on 23 October 2017).

47. Schroeder, W.; Oliva, P.; Giglio, L.; Csiszar, I.A. The New VIIRS 375m active fire detection data product: Algorithm description and initial assessment. Remote Sens. Environ. 2014, 143, 85-96. [CrossRef]

48. Oliva, P.; Schroeder, W. Assessment of VIIRS $375 \mathrm{~m}$ active fire detection product for direct burned area mapping. Remote Sens. Environ. 2015, 160, 144-155. [CrossRef]

49. Giglio, L.; Schroeder, W.; Justice, C.O. The collection 6 MODIS active fire detection algorithm and fire products. Remote Sens. Environ. 2016, 178, 31-41. [CrossRef]

50. Boschetti, L.; Flasse, S.P.; Brivio, P.A. Analysis of the conflict between omission and commission in low spatial resolution dichotomic thematic products: The Pareto Boundary. Remote Sens. Environ. 2004, 91, 280-292. [CrossRef]

51. Chuvieco, E.; Opazo, S.; Sione, W.; del Valle, H.; Anaya, J.; Di Bella, C.; Cruz, I.; Manzo, L.; López, G.; Mari, N.; et al. Global Burned-Land Estimation in Latin America Using Modis Composite Data. Ecol. Appl. 2008, 18, 64-79. [CrossRef] [PubMed]

52. Padilla, M.; Stehman, S.V.; Ramo, R.; Corti, D.; Hantson, S.; Oliva, P.; Alonso-Canas, I.; Bradley, A.V.; Tansey, K.; Mota, B.; et al. Comparing the accuracies of remote sensing global burned area products using stratified random sampling and estimation. Remote Sens. Environ. 2015, 160, 114-121. [CrossRef]

53. Boschetti, L.; Roy, D.P.; Justice, C.O. International Global Burned Area Satellite Product Validation Protocol Part I-Production and standardization of validation reference data. 2009; Unpublished Data.

54. Blaschke, T. Object based image analysis for remote sensing. ISPRS J. Photogramm. Remote Sens. 2010, 65, 2-16. [CrossRef]

55. Pereira, A.A.; Libonati, R.; Oom, D.; Carvalho, L.M.T.; Pereira, J.M.C. Avaliação de técnicas de compósitos multitemporais em imagens PROBA-V para o mapeamento de áreas queimadas. In Proceedings of the Simpósio Brasileiro de Sensoriamento Remoto, Santos, Brazil, 25-29 April 2015; Volume XVIII.

56. Barbosa, P.M.; Grégoire, J.M.; Pereira, J.M.C. An algorithm for extracting burned areas from time series of AVHRR GAC data applied at a continental scale. Remote Sens. Environ. 1999, 69, 253-263. [CrossRef]

57. McKeeman, W.M. Peephole optimization. Commun. ACM 1965, 8, 443-444. [CrossRef]

58. Karatzoglou, A.; Meyer, D.; Hornik, K. Support Vector Algorithm in R. J. Stat. Softw. 2006, 15, 1-28. [CrossRef]

59. Cao, X.; Chen, J.; Matsushitas, B.; Imura, H.; Wang, L. An automatic method for burn scar using vector machines. Int. J. Remote Sens. 2009, 30, 577-594. [CrossRef]

60. Dragozi, E.; Gitas, I.Z.; Stavrakoudis, D.G.; Theocharis, J.B. Burned area mapping using support vector machines and the FuzCoC feature selection method on VHR IKONOS imagery. Remote Sens. 2014, 6, 12005-12036. [CrossRef]

61. Chang, C.; Lin, C. LIBSVM: A Library for Support Vector Machines. ACM Trans. Intell. Syst. Technol. 2011, 2, 1-27. [CrossRef]

62. Wilks, D.S. Statistical Methods in the Atmospheric Sciences; Academic Press: Cambridge, UK, 2011; Volume 100, ISBN 0123850223.

63. Binaghi, E.; Brivio, P.A.; Ghezzi, P.; Rampini, A. A fuzzy set accuracy assessment of soft classification. Pattern Recognit. Lett. 1999, 20, 935-948. [CrossRef]

64. Padilla, M.; Stehman, S.V.; Litago, J.; Chuvieco, E. Assessing the temporal stability of the accuracy of a time series of burned area products. Remote Sens. 2014, 6, 2050-2068. [CrossRef]

65. Hamed, K.H.; Ramachandra Rao, A. A modified Mann-Kendall trend test for autocorrelated data. J. Hydrol. 1998, 204, 182-196. [CrossRef]

66. Giglio, L.; van der Werf, G.R.; Randerson, J.T.; Collatz, G.J.; Kasibhatla, P. Global estimation of burned area using MODIS active fire observations. Atmos. Chem. Phys. Discuss. 2005, 5, 11091-11141. [CrossRef]

67. Pereira, A.A.; Fabrício Rodrigues Teixeira, R.L.; Melchiori, E.A.; Carvalho, L.M.T. Avaliação de índices espectrais para identificação de áreas queimadas no cerrado utilizando dados LandSat TM. Rev. Bras. Cartogr. 2016, 8, 1665-1680.

68. White, J.D.; Ryan, K.C.; Key, C.C.; Running, S.W. Remote Sensing of Forest Fire Severity and Vegetation Recovery. Int. J. Wildland Fire 1996, 6, 125-136. [CrossRef]

69. Libonati, R.; DaCamara, C.C.; Pereira, J.M.C.; Peres, L.F. On a new coordinate system for improved discrimination of vegetation and burned areas using MIR/NIR information. Remote Sens. Environ. 2011, 115, 1464-1477. [CrossRef] 
70. Libonati, R.; DaCamara, C.C.; Pereira, J.M.C.; Peres, L.F. Retrieving middle-infrared reflectance using physical and empirical approaches: Implications for burned area monitoring. IEEE Trans. Geosci. Remote Sens. 2012, 50, 281-294. [CrossRef]

71. DaCamara, C.C.; Libonati, R.; Ermida, S.L.; Calado, T.J. A User-Oriented Simplification of the (V, W) Burn-Sensitive Vegetation Index System. IEEE Geosci. Remote Sens. Lett. 2016, 13, 1822-1825. [CrossRef]

72. Trigg, S.; Flasse, S. An evaluation of different bi-spectral spaces for discriminating burned shrub-savannah. Int. J. Remote Sens. 2001, 22, 2641-2647. [CrossRef]

73. Pereira, J.M.; Sa, A.C.L.; Sousa, A.M.O.; Silva, J.M.N.; Santos, T.N.; Carreiras, J.M.B. Spectral characterisation and discrimination of burnt areas. Remote Sens. Large Wildfires 1999, 123-138. [CrossRef]

74. Miranda, H.S.; Sato, M.N. Efeitos do fogo na vegetação lenhosa do Cerrado. In Cerrado Ecologia E Caracterização; Ministério do Meio Ambiente: Brasília, Brazil, 2004; pp. 66-123. ISBN 9789728669485.

(C) 2017 by the authors. Licensee MDPI, Basel, Switzerland. This article is an open access article distributed under the terms and conditions of the Creative Commons Attribution (CC BY) license (http:/ / creativecommons.org/licenses/by/4.0/). 